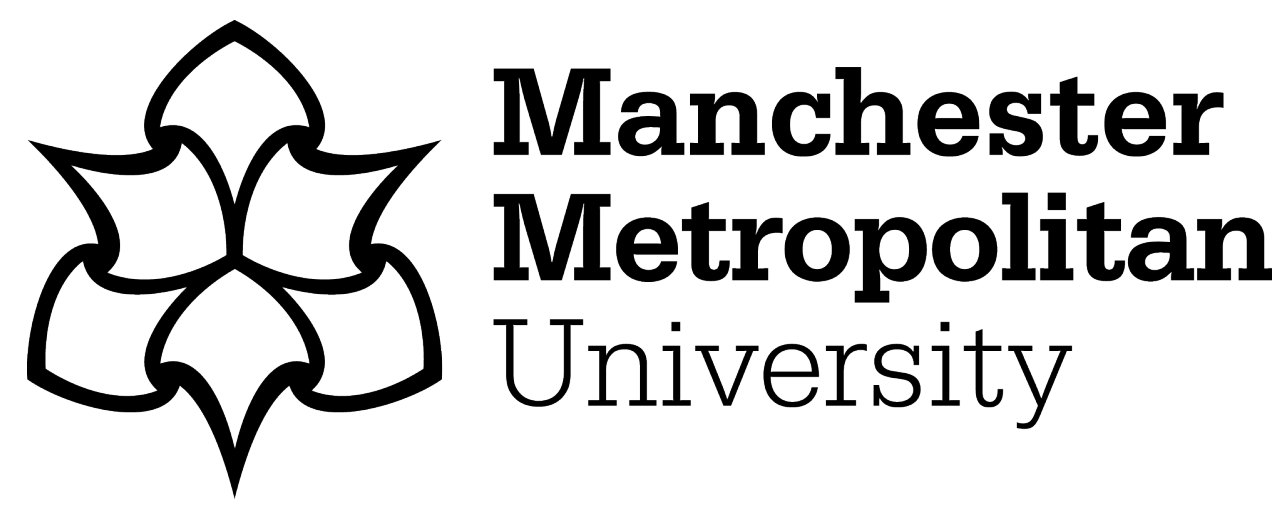

O'Dowd, Dawn N, Bostock, Emma L, Smith, Dave ORCID logoORCID: https://orcid.org/0000-0002-3711-4381, Morse, Christopher I, Orme, Paul and Payton, Carl J (2021) Psychological parameters impact health-related quality of life in mental and physical domains in adults with muscular dystrophy. Neuromuscular Disorders, 31 (4). pp. 328-335. ISSN 0960-8966

Downloaded from: https://e-space.mmu.ac.uk/627338/

Version: Accepted Version

Publisher: Elsevier

DOI: https://doi.org/10.1016/j.nmd.2021.01.007

Usage rights: Creative Commons: Attribution-Noncommercial-No Derivative Works 4.0

Please cite the published version 


\section{Psychological parameters impact health-related quality of life in mental and physical domains in adults with muscular dystrophy}

Dawn N. O'Dowd ${ }^{1}$ Emma L. Bostock ${ }^{2}$, Dave Smith ${ }^{1}$, Christopher I. Morse ${ }^{1}$, Paul Orme ${ }^{3}$, and Carl J. Payton ${ }^{1}$

${ }^{1}$ Research Centre for Musculoskeletal Science \& Sports Medicine, Department of Sport and Exercise Sciences, Manchester Metropolitan University, Manchester, United Kingdom

${ }^{2}$ Musculoskeletal Physiology Research Group, School of Science and Technology, Nottingham Trent University, Nottingham, United Kingdom

${ }^{3}$ The Neuromuscular Centre, Winsford, Cheshire, United Kingdom

Corresponding Author: Dawn N. O'Dowd, Department of Sport and Exercise Sciences, All Saints Building, Manchester Metropolitan University, Manchester, M15 6BH, United Kingdom. Email: D.ODowd@mmu.ac.uk 


\section{Abstract}

The impacts of potentially treatable psychological parameters on quality of life are relatively unreported in adults with Facioscapulohumeral, Becker and Limb-girdle muscular dystrophy. The purpose of this study was to compare quality of life, psychological parameters, and physical function between adults with muscular dystrophy and controls, and to examine relationships among these parameters in muscular dystrophy. Twenty-one adults with muscular dystrophy ( $n=7$ Becker, $n=8$ Facioscapulohumeral, $n=6$ Limb-girdle) and ten agematched controls participated. Outcome measures were health-related quality of life, depressive symptoms, trait anxiety, self-esteem, physical self-worth and six-minute walk distance. Quality of life scores were lower in the muscular dystrophy groups than the control $(p<.05)$. Depressive symptoms had the greatest association with quality of life in the Mental Health domain $(r=-.89, p<.001)$. Depressive symptoms also had the most associations with quality of life ( 7 of 10 domains), followed by trait anxiety (6 of 10 domains), physical selfworth ( 5 of 10 domains), self-esteem (4 of 10 domains) and six-minute walk distance ( 3 of 10 domains). Psychological parameters and, to a lesser extent, physical function impact quality of life in muscular dystrophy. This study provides a rationale to include psychological assessment and treatment within muscular dystrophy healthcare.

Keywords: Mental health, Depression, Self-esteem, Anxiety, Physical function 


\section{Introduction}

Muscular dystrophy (MD) is a collection of inherited neuromuscular disorders caused by mutations in various genes [1]. Such mutations result in either a problem with, or lack of, one of the proteins within muscle cells[2]. Facioscapulohumeral (FSHD), Becker (BMD) and Limbgirdle (LGMD) MD are associated with defects in the proteins of the sarco-glycan complex [35]. Despite variations in genotype between $M D$ conditions and condition-specific presentations of physical impairment, all MDs cause progressive reductions in muscle strength and a worsening ability to perform daily functional tasks such as walking [6].

Low health-related quality of life (QoL) scores have consistently been reported in individuals with impaired muscle function [7]. QoL scores in MD populations however are varied. Studies have reported lower QoL scores in children and adolescents with Duchenne MD [8,9], a combined group of children and adults with Duchenne MD, BMD and LGMD [10] and adults with FSHD $[11,12]$ compared to controls or normative reference values, in QoL domains related to both physical and mental aspects. Other studies have found lower QoL_scores in physical domains compared to controls or normative reference values but not in the mental health domain of QoL [13-16]. These latter findings have been shown in Duchenne MD [1315] and recently in adults with LGMD and Duchenne MD [16], and they reflect the Disability Paradox Phenomenon [17] whereby self-reported QoL scores are high despite substantial physical disability.

The relationship between $\mathrm{MD}$ and $\mathrm{QoL}$ is evidently complicated. A range of disease-specific variables have been found to be associated with QoL scores in physical domains in individuals with MD. These include age $[8,12,18]$, condition severity $[12,19]$, functional status [9], wheelchair dependence/use [20] and age at disease onset [18]. Other parameters are 
associated with both physical and mental domains of QoL in MD, including pain $[12,16,21]$ and fatigue $[16,20,22]$. Whilst disease-specific parameters, such as age at disease onset, provide insight into QoL they cannot be treated, whilst aspects like pain and fatigue can be treated. It is important to establish the impact of other potentially treatable parameters on QoL scores, such as psychological aspects, as they may be as, if not more, important than physical ones.

Symptoms of depression and anxiety are known to negatively affect QoL scores in medical conditions such as diabetes [23-26], but their impact on QoL in FSHD, LGMD and BMD remain relatively unreported. There is however some evidence that QoL scores are affected by psychological parameters in MD. In adults with Duchenne MD, a negative relationship was found between anxiety and both physical and mental domains of QoL, and between depression and physical domains of QoL [27]. In adults with LGMD, a moderate negative association was found between depression and overall QoL [18], but details regarding which QoL domains were not provided. In adults with FSHD, depressive symptoms were shown to be negatively related to both physical and mental domains of QoL [12]. The impact of depressive symptoms on QoL is yet to be reported in BMD and the impact of anxiety on QoL remains unreported in FSHD, BMD and LGMD.

In addition to depression and anxiety, a person's self-perceptions play an important role in their mental wellbeing [28]. Self-esteem, a general positive or negative self-regard [29], has been shown to positively impact QoL scores in adults with multiple sclerosis [30], women with cervical cancer [31] and women with fibromyalgia [32]. The impact of self-perceptions on QoL has received little attention in MD. One study measured general self-efficacy in adults with Duchenne MD, FSHD and BMD and found small to moderate associations with QoL [16], but 
no assessments of self-esteem or physical self-worth have yet been conducted in adults with MD.

Muscle deterioration is a characteristic feature of FSHD, LGMD and BMD [6]. It is therefore unsurprising that maximum knee extensor strength was found to be moderately associated with QoL scores in the Physical Function and Social Function domains in adults with BMD [16], and that hip flexor muscle strength was moderately associated with overall QoL scores in adults with LGMD [18]. These findings highlight the impact of physical disease progression on QoL scores in MD, but whether physical function has a greater impact on QoL scores than psychological health in adults with MD is undetermined. The six-minute walk (6MW) test is an established and advocated outcome measure of physical function in MD [33], which may provide insight into the effect that physical function has on QoL scores in MD.

This study will contribute to a greater understanding of how QoL scores in adults with FSHD, BMD and LGMD differs from non-dystrophic adults, and it will highlight parameters that impact QoL in adults with MD to inform future healthcare.

\subsection{Aims}

1) To compare QoL scores of adults with FSHD, BMD and LGMD to a non-dystrophic agematched control group. 2) To compare between the groups for psychological parameters (depressive symptoms, trait anxiety, self-esteem, and physical self-worth) and physical function (6MW distance). 3) To examine associations between QoL scores and psychological parameters and between QoL scores and physical function in adults with MD. 


\section{Materials and Methods}

\subsection{Participants}

Twenty-one adults diagnosed with one of three variants of $\mathrm{MD}(\mathrm{n}=7 \mathrm{BMD}, \mathrm{n}=8 \mathrm{FSHD}$ and $\mathrm{n}=6 \mathrm{LGMD}$ ) took part, along with ten age-matched control adults. The participant characteristics of the control group, MD sub-groups (BMD, FSH and LGMD) and a pooled average for MD are described in Table 1. All participants were of sound intellectual status. Control participants were free from any known health problems, illnesses or injuries and were recruited from the general population. MD participants were in otherwise good health without any uncontrolled co-morbidity or cardiac issues and were able to walk at least seven metres with or without assistive walking devices. They were recruited from a Neuromuscular Centre (Cheshire, UK), where they receive physiotherapy (weekly, bi-weekly or monthly) to maintain function. All participants self-reported that they were un-trained and did not undertake more than one hour of intense physical activity or three hours of low-moderate physical activity per week. The Manchester Metropolitan University Ethics Committee granted ethical approval and all participants provided written informed consent before participating.

\subsection{Procedures}

Participant characteristics, which were age (years), stature $(\mathrm{cm})$, body mass $(\mathrm{kg})$, muscle strength (N.m) and work status, were collected initially. Stature and body mass were measured using a stadiometer and digital scales, and isometric maximum voluntary contraction torque during knee flexion and knee extension was measured using an isokinetic dynamometer (Cybex Norm, Cybex International Inc., NY, USA), as described in a previous study [34]. Participants then completed a $6 \mathrm{MW}$ test and five questionnaires, which assessed QoL_scores, symptoms 
of depression, trait anxiety, self-esteem, and physical self-worth. Each questionnaire was explained to participants, and investigators were available if participants required assistance.

\subsubsection{Six-Minute Walk Test}

Sixteen MD participants completed a $6 \mathrm{MW}$ test $(\mathrm{n}=6 \mathrm{FSHD}, \mathrm{n}=4 \mathrm{BMD}, \mathrm{n}=6 \mathrm{LGMD}$ ). Five participants did not complete this test as they expressed a fear of falling. An adapted version of the protocol set out by the American Thoracic Society [35] was utilised. Participants were instructed to walk at their self-selected pace, instead of as far as possible as prescribed by the American Thoracic Society [35], due to safety concerns in the current population.

The test took place along a straight corridor. Each end of the $25 \mathrm{~m}$ course was marked with tape on the ground. Participants were instructed to turn clockwise at each end and walk in a straight path. One investigator walked behind the participant, keeping at least a $2 \mathrm{~m}$ distance, to provide encouragement (in accordance with The American Thoracic Society guidelines) and assess fall risk. Participants used their walking aids during the test if they typically used them to walk for a period of six minutes. After five minutes' seated rest, participants were readied at the start line and the timer was started once they began to walk. After six minutes, participants were seated and the distance walked was measured.

\subsubsection{Quality of Life -The 36-item Short Form Health Survey Version 2}

The 36-item Short Form Health Survey is a self-report generic survey that covers 36 questions on functional health and wellbeing to assess QoL from the participant's perspective [36]. Each question has a multiple-choice scale on which participants rate themselves. The answers are summarised into four physical domains (Physical Function, Role Physical, Bodily Pain and General Health), four mental health domains (Vitality, Social Function, Role Emotion and 
Mental Health) and two component health scores (Physical Component Score and Mental Component Score). Scores can range between 0 - 100 on each domain. Higher scores indicate a better QoL score. The scale has excellent test-retest reliability with intraclass correlation coefficients (ICC) above .80 [36].

\subsubsection{Depressive Symptoms -The Beck Depression Inventory}

The Beck Depression Inventory is a 21 question, multiple choice, self-report questionnaire that measures the severity of depressive symptoms. It has excellent reliability, with a testretest ICC of .96 [37]. The questionnaire includes items relating to symptoms of depression and participants score each question from 0 (least) to 3 (most), with a total sum score indicating overall depressive symptom severity from 0-63. Higher scores indicate increased severity of depressive symptoms. The cut scores for The Beck Depression Inventory are as follows: $\leq 10$ indicates zero to minimal depressive symptoms, 11-20 indicates mild depressive symptoms, $21-30$ indicates moderate depressive symptoms, and $\geq 31$ indicates severe depressive symptoms.

\subsubsection{Trait Anxiety - State-Trait Anxiety Inventory}

The trait sub-scale of the State-Trait Anxiety Inventory [38] is a 20 question multiple choice self-report questionnaire that measures trait-anxiety (a predisposition to long-lasting and persistent feelings of anxiety that are not restricted to particular circumstances). The trait sub-scale has excellent test-retest reliability, with a reported ICC of .86 [38]. The scale includes statements for participants to score between 1 (not at all) and 4 (very much so). Total scores can range between $20-80$, with higher scores indicating more severe anxiety symptoms. 


\subsubsection{Self-Esteem - Rosenberg Self-Esteem Scale}

The Rosenberg Self-Esteem Scale is a measure of global self-esteem [39]. The scale has excellent reported reliability, with an ICC of .90 [40]. It has 10 questions that assess both the positive and negative feelings towards the self. Participants rate themselves on a 4-point Likert scale, with total scores ranging from 10 to 40 . The higher the score the higher the selfesteem.

\subsubsection{Physical Self-worth -The Physical Self-Perception Profile}

The Physical Self-Perception Profile is a reliable (test-retest ICCs between .74 and .91) selfreport questionnaire [41] that comprises thirty questions that assess physical self-worth in the global domain and self-perceptions in four sub-scales: sport competence, body attractiveness, physical strength and physical conditioning and exercise. An additional eight questions relate to perceived importance of the four sub-scales and provide a measure of importance attached by the participant to the respective sub-scale.

\subsection{Data Analysis}

Each questionnaire was scored according to the relevant manual. QoL data were scored using Health Outcomes Scoring Software 4.5 (Quality Metric Health Outcomes ${ }^{\mathrm{TM}}$, Lincoln, United Kingdom).

Data are presented as means and standard deviations for the control group, pooled MD group and each MD sub-group. Statistical analysis is between the MD subgroups (FSHD, LGMD and $\mathrm{BMD}$ ) and the control group, and because no difference in primary outcome measures were 
found between the MD sub-groups, statistical analysis was also conducted between the pooled MD group and the control group.

\subsection{Statistical Analysis}

All statistical analysis was completed using IBM SPSS Statistics 22 software. The critical level of significance was set at $p<.05$.

Age, stature and body-mass data met the parametric assumptions. For these data, one-way ANOVAs were conducted to test for differences between the three MD subgroups and the control group, and independent t-tests were conducted to test for differences between the pooled MD group and the control group.

$6 \mathrm{MW}$ data were not normally distributed and all questionnaire data were interpreted as nonparametric. For these data, Kruskall Wallis tests were conducted to test for differences between the three MD subgroups and the control group, with Mann-Whitney $U$ post-hoc pairwise comparisons where appropriate. To test for differences between the pooled MD group and the control group, Mann-Whitney $U$ tests were conducted. For $6 \mathrm{MW}$ data, $\mathrm{n}=16$ $\mathrm{MD}$ and 10 control participants to compare between the control and pooled MD group, and $\mathrm{n}=6 \mathrm{FSHD}, \mathrm{n}=4 \mathrm{BMD}, \mathrm{n}=6 \mathrm{LGMD}$ and $\mathrm{n}=10$ control to compare between the MD subgroups and the control group.

To examine associations, Spearman's Rank correlations were performed between each domain of quality of life and each outcome variable. As no difference was found between the three MD subgroups for the primary outcome variables, the FSHD, BMD and LGMD subgroups were merged into one MD group for correlational analysis. Correlations were interpreted 
such that $\leq .40=$ small, $.41-.60=$ moderate, .61 to $.79=$ large and $\geq .80=$ very large [42]. For $6 \mathrm{MW}$ data, $\mathrm{n}=16 \mathrm{MD}$ participants for correlational analysis.

\section{Results}

\subsection{Participants}

No significant difference in age, stature or body mass was found between the MD subgroups or between any MD group and the control group (Table $1 ; p>.05$ ).

\subsection{Quality of Life}

QoL scores between the groups are presented in Table 2 .

No difference was found among the MD subgroups (FSHD, BMD and LGMD) in any QoL domain $(p>.05)$. A main effect of group was found in the following QoL domains: Physical Function ( $p \leq .001)$, Role Physical $(p \leq .001)$, Bodily Pain $(p=.019)$, General Health $(p=.026)$, Vitality $(p=.005)$, Social Function $(p=.002)$, Role Emotion $(p=.036)$ and Physical Component Score $(p \leq .001)$. No difference was found in the Mental Health domain or Mental Component Score $(p>.05)$. Post hoc comparisons found that QoL scores in the following domains were lower in each MD subgroup compared to the control group: Physical Function (FSHD,LGMD, BMD: $p \leq .001$ ), Role Physical (FSHD, LGMD, BMD: $p \leq .001$ ), General Health (FSHD: $p=.044$, LGMD: $p=.008$, BMD: $p=.027$ ), Vitality (FSHD: $p=.006$, LGMD: $p=.002$, BMD: $p=.022$ ), Social Function (FSHD: $p=.005$, LGMD: $p=.002$, BMD: $p \leq .001$ ), Role Emotion (FSHD: $p=.011$, LGMD: $p=.024$, BMD: $p=.022$ ) and Physical Component Score (FSHD, LGMD, BMD: $p \leq .001)$. In the Bodily Pain domain, scores were lower in the FSHD $(p=.002)$ and LGMD $(p=.044)$ groups than the control group. 
In the pooled MD group, QoL scores were lower than the control group in the Physical Function ( $p \leq .001)$, Role Physical $(p \leq .001)$, Bodily Pain $(p=.002)$ General Health $(p=.002)$, Vitality $(p \leq .001)$, Social Function $(p \leq .001)$, Role Emotion $(p=.003)$ and Physical Component Score $(p \leq .001)$. No difference was found in the Mental Health domain or Mental Component Score $(p>.05)$.

\subsection{Psychological Parameters and 6MW}

Psychological parameters and 6MW distance are presented in Table 3.

No difference in any of these measures were found between the MD subgroups $(p>.05)$. A main effect of group was found in physical self-worth $(p=.006)$ and $6 \mathrm{MW}$ distance $(p=.005)$. Depressive symptoms, trait anxiety and self-esteem did not differ between the groups ( $p$ > .05). Post hoc comparisons found that physical self-worth was lower in the FSHD $(p=.016)$, LGMD ( $\leq .001)$ and BMD ( $p=.026)$ groups than the control group. Distance walked during the $6 \mathrm{MW}$ test was lower in the BMD $(p=.005)$ and LGMD $(p=.009)$ groups than the control group.

In the pooled MD group, depressive symptoms were greater $(p=.011)$, and physical selfworth $(p \leq .001)$ and $6 \mathrm{MW}$ distance $(p \leq .001)$ were lower than the control group. Trait anxiety and self-esteem did not differ between the groups ( $p>.05)$.

\subsection{Quality of Life Correlations}

Correlation coefficients showing the strength of association between QoL scores and psychological parameters/6MW in pooled MD participants are presented in Table 4. 
Depressive symptoms were negatively associated with seven of the ten QoL domains. Moderate associations were found between depressive symptoms and Bodily Pain $(r=-.45, p$ $=.041)$, General Health $(r=-.43, p=.049)$ and Vitality $(r=-.45, p=.043)$. Large associations were found between depressive symptoms and Social Function ( $r=-.66, p \leq .001$ ), and between depressive symptoms and Role Emotion ( $r=-.78, p \leq .001)$. Very large associations were found between depressive symptoms and Mental Health $(r=-.89, p \leq .001)$ and the Mental Component Score $(r=-.87, p \leq .001)$. Physical Function, Role Physical and the Physical Component Score were not associated with depressive symptoms ( $p>.05)$.

Trait anxiety was negatively correlated with numerous QoL domains. Trait anxiety was moderately correlated with Role Physical $(r=-.44, p=.045)$ and Vitality $(r=-.48, p=.027)$. Trait anxiety was largely associated with Social Function ( $r=-.74, p \leq .001)$, Role Emotion ( $r$ $=-.85, p \leq .001)$, Mental Health $(r=-.81, p \leq .001)$ and the Mental Component Score $(r=-.82$, $\mathrm{p} \leq .001)$

Self-esteem was positively associated with various QoL domains. Self-esteem had a moderate association with Social Function $(r=.47 ; p=.033)$, and Role Emotion $(r=.68, p \leq .001)$. Mental Health $(r=.77, p \leq .001)$ and the Mental Component Score $(r=.74, p \leq .001)$ were largely associated with self-esteem. No other QoL domains were associated with self-esteem ( $p$ > $.05)$.

Physical self-worth was positively correlated with QoL in the Role Physical $(r=.49, p=.033)$, General Health $(r=.50, p=.029)$, Vitality $(r=.52, p=.023)$, Mental Health $(r=.58, p=.009)$ and Mental Component Score $(r=.55, p=.014)$. 
$6 \mathrm{MW}$ distance was moderately correlated with the Vitality domain $(r=.51 ; p=.043)$ and highly correlated with Physical Function $(r=.65 ; p=.007)$ and the Physical Component Score $(r=.64 ; p=.008)$. No other domains were associated with 6MW distance $(p>.05)$.

\section{Discussion}

This study presents a comparison of QoL scores, psychological parameters and 6MW distance between adults with MD to matched control adults, and factors that impact QoL in adults with MD are identified. QoL scores and physical self-worth were lower in adults with FSHD, BMD and LGMD than controls, and 6MW distance and depressive symptoms were poorer in the MD group than controls. Psychological parameters and 6MW distance impact QoL, with depressive symptoms showing the greatest and most consistent impact on QoL.

\subsection{Quality of Life}

QoL scores were poorer in various physical and mental domains for the MD groups compared to the control group, except in the Mental Health domain and the Mental Component Score. This finding agrees with previous research that reported no difference in QoL scores in the Mental Health domain in children and adults with Duchenne MD [13-15], and with a more recent study that found no difference in the Mental Component Score of QoL between adults with Duchenne MD, FSHD, BMD and LGMD compared to control adults [16].

The findings in this study reflect the Disability Paradox [17], as QoL scores in the Mental Health domain was comparable across all groups despite physical disability. This finding had until recently only been reported in Duchenne MD [13-15], which had prompted suggestions that those with Duchenne MD may cope better than those with other types of MD because of earlier onset and limited experience of life without MD [13]. The MD participants in this study 
were recruited from a Neuromuscular Centre, where they receive regular physiotherapy and other support. This association may support the development of coping mechanisms, or perhaps it negates some of the factors that impact QoL in the Mental Health domain. Despite comparable QoL scores in the Mental Health domain, QoL scores were poorer than controls in seven other domains for each MD subgroup and eight other domains for the FSHD and LGDM group. Therefore, improving QoL scores in adults with FSHD, BMD and LGMD should be at the forefront of healthcare.

\subsection{Psychological Parameters and Physical Function}

Differences in psychological parameters and physical function were evident between the MD and control adults. $6 \mathrm{MW}$ distance was significantly reduced compared to the control group in both the LGMD and BMD groups, and depressive symptoms were significantly greater in the pooled MD group than the control group. No difference in trait anxiety or self-esteem was evident between the MD groups and the control group, but physical self-worth was considerably reduced in all MD groups compared to the control group. Self-esteem is a multidimensional construct [43] said to be influenced by various domain levels of self-worth such as physical, academic, cognitive and social. The current findings highlight that adults with FSHD, BMD and LGMD exhibit reduced physical self-worth but comparable self-esteem to control participants. The implications of this are twofold: 1) increasing physical self-worth may improve global self-esteem for adults with MD and 2) increasing self-worth in other areas could limit the impact of poor physical self-worth on self-esteem in MD. It is important to note that a large proportion of the MD participants in this study (91\%) were in, or had previously been in, paid employment, and sociodemographic factors such as work status may influence selfesteem and other psychological parameters in this population. 


\subsection{Associations with Quality of Life}

QoL scores were consistently associated with psychological parameters including depressive symptoms, trait anxiety, self-esteem and physical self-worth. The size of associations was typically large with mental domains of QoL and moderate with physical domains of QoL. Depressive symptoms showed the most consistent association with QoL (7 of 10 domains), followed by trait anxiety ( 6 of 10 domains), physical self-worth (5 of 10 domains) and selfesteem (4 of 10 domains). Depressive symptoms also showed the largest association with QoL scores in the Mental Health domain, followed by trait anxiety in the Role Emotion domain. These results agree with findings in patients with other medical conditions such as diabetes [23-26], fibromyalgia [32] and cervical cancer [31]. Previous research has reported a small and a moderate relationship between depression and QoL scores in adults with FSHD [12] and adults with LGMD[18], respectively, but this is the first study to demonstrate associations between QoL and self-esteem, QoL and physical self-worth and QoL and trait anxiety in adults with FSHD, BMD and LGMD.

It is clear that symptoms of depression, trait anxiety, self-esteem and physical self-worth are important determinants of QoL scores in adults with FSHD, BMD and LGMD. Importantly, depressive symptoms, trait anxiety and physical self-worth impact the physical domains of QoL as well as mental domains. Therefore, despite the progressive physical nature of MD the management of psychological health may prove highly beneficial in improving QoL across the board in this population.

Despite reduced physical function being one of the main characteristics of MD, the impact of 6MW distance on QoL scores was limited to three domains. 6MW distance was associated with QoL in Physical Function, Vitality and the Physical Component Score. This finding is 
similar to previous research in BMD [16] which found knee extension muscle strength was associated with QoL in the Physical Function and Social Function domains only.

\subsection{Clinical Implications}

Psychological parameters and 6MW distance impact QoL in adults with MD. Interventions that target either, or preferably both, of these areas could be highly advantageous for improving QoL in this population. Exercise may be a valuable intervention given that it targets both physical and mental health. Future research should seek to establish if exercise can improve psychological and physical parameters in adults with MD and, in turn, improve QoL scores.

\subsection{Study Limitations}

All MD participants were recruited from a Neuromuscular Centre, where they regularly receive physiotherapy and other support services. This support network may account for the similarities in Mental Health QoL found in this study between the MD and control participants, compared to other studies that reported differences between these groups. However, this finding does highlight the importance of such support networks on QoL in this population. It is also important to acknowledge that a reduced number of MD participants completed the $6 \mathrm{MW}$ test because they expressed a fear of falling. As such, there is less confidence in the findings regarding the $6 \mathrm{MW}$ test than the psychological parameters in this study, as sixteen participants completed the test compared to twenty-one in the other parameters. Further research with a larger sample of participants would therefore be advantageous to substantiate these findings. 


\section{Conclusion}

In adults with FSHD, BMD and LGMD, QoL scores in mental and physical domains were lower compared to age-matched controls. Symptoms of depression and anxiety have a negative impact on mental and physical domains of QoL in adults with MD. Additionally, physical selfworth, self-esteem and, to a lesser extent, $6 \mathrm{MW}$ distance are important determinants of QoL in this population. This study provides a rationale for the assessment and treatment of psychological health alongside physical health for adults with MD. Interventions that target psychological health should be employed, especially where QoL scores are low. 


\section{Acknowledgements}

The authors gratefully acknowledge the contribution of staff, patients, and patients' family members at the Neuromuscular Centre.

\section{Funding Source}

This research did not receive any specific grant from funding agencies in the public, commercial, or not-for-profit sectors.

\section{Declarations of Interest}

None

\section{Authors' Contributions}

All authors contributed to the design of this paper. DO and EB contributed to the data collection, analysis and the writing of the manuscript. $\mathrm{CP}, \mathrm{CM}$ and DS supervised the project. PO contributed to participant identification and recruitment. All authors critically revised the manuscript and approved the final version. 


\section{References}

[1] Huml RA. Muscular dystrophy: A concise guide. Springer; 2015.

[2] Cohn RD, Campbell KP. Molecular basis of muscular dystrophies. Muscle \& Nerve 2000; 23: 1456 - 71. https://doi.org/10.1002/1097-4598(200010)23:10<1456::AIDMUS2>3.0.CO;2-T.

[3] Emery AEH. The muscular dystrophies. The Lancet 2002; 359: 687-95.

[4] Tawil R, Van Der Maarel SM, Tapscott SJ. Facioscapulohumeral dystrophy: the path to consensus on pathophysiology. Skeletal Muscle 2104; 4: 12-27.

[5] Murphy AP, Straub V. The classification, natural history and treatment of the limb girdle muscular dystrophies. Journal of Neuromuscular Diseases 2 2015; s2: s7-s19. https://doi.org/10.3233/JND-150105.

[6] Mercuri E, Muntoni F. Muscular dystrophies. The Lancet 2013; 381: 845-60. http://doi.org/10.1016/S0140-6736(12)61897-2.

[7] Graham CD, Rose MR, Grunfeld EA, Kyle SD, Weinman J. A systematic review of quality of life in adults with muscle disease. Journal of Neurology 2011; 258: 1581-92. https://doi.org/10.1007/s00415-011-6062-5.

[8] Uzark K, King E, Cripe L, Spicer R, Sage J, Kinnett K, et al. Health-related quality of life in children and adolescents with Duchenne muscular dystrophy. Pediatrics 2012; 130: 1559-66. https://doi.org/10.1542/peds.2012-0858.

[9] Lue YJ, Chen SS, Lu YM. Quality of life of patients with Duchenne muscular dystrophy: from adolescence to young men. Disability and Rehabilitation 2016; 39: 1-6. https://doi.org/10.1080/09638288.2016.1196398.

[10] Grootenhuis MA, de Boone J, van der Kooi AJ. Living with muscular dystrophy: health related quality of life consequences for children and adults. Health and Quality of Life Outcomes 2007; 5: 31-38. https://doi.org/10.1186/1477-7525-5-31.

[11] Winter Y, Schepelmann K, Spottke AE, Claus D, Grothe C, Schroder R, et al. Health-related quality of life in ALS, Myasthenia Gravis and Facioscapulohumeral muscular dystrophy. Journal of Neurology 2010; 257: 1473-81. https://doi.org/10.1007/s00415-010-5549-9.

[12] Padua L, Aprile I, Frusciante R, lannaccone E, Rossi M, Renna R, et al. Quality of life and pain in patients with Facioscapulohumeral muscular dystrophy. Muscle \& Nerve 2009; 40: 200-05. https://doi.org/10.1002/mus.21308.

[13] Kohler M, Clarenbach CF, Böni L, Brack T, Russi EW, Bloch KE. Quality of life, physical disability, and respiratory impairment in Duchenne muscular dystrophy. American Journal of Respiratory and Critical Care Medicine 2005; 172: 1032-36. https://doi.org/10.1164/rccm.200503-3220C. 
[14] Pangalila RF, van den Bos GA, Bartels B, Bergen MP, Kampelmacher MJ, Stam HJ, et al. Quality of life of adult men with Duchenne muscular dystrophy in The Netherlands: Implications for care. Journal of Rehabilitation Medicine 2015; 47: 161-66. https://doi.org/10.2340/16501977-1898.

[15] Elsenbruch S, Schmid J, Lutz S, Geers B, Schara U. Self-reported quality of life and depressive symptoms in children, adolescents, and adults with Duchenne muscular dystrophy: A cross-sectional survey study. Neuropediatrics 2013; 44: 257-64. https://doi.org/10.1055/s-0033-1347935.

[16] Jacques MF, Stockley RC, Onambele-Pearson GL, Reeves ND, Stebbings GK, Dawson EA, et al. Quality of life in adults with muscular dystrophy. Health and Quality of Life Outcomes 2019; 17: 121-31. https://doi.org/10.1186/s12955-019-1177-y.

[17] Albrecht GL, Devlieger PJ. The disability paradox: high quality of life against all odds. Social Science \& Medicine 1999; 48: 977-88.

[18] Peric M, Peric S, Stevanovic J, Milovanovic S, Basta I, Nikolic A, et al. Quality of life in adult patients with limb-girdle dystrophies. Acta Neurologica Belgica 2018: 118: 243-50. https://doi.org/10.1007/s13760-017-0857-9.

[19] Otto C, Steffensen BF, Hojberg AL, Barkmann C, Rahbek J, Ravens-Sieberer U, et al. Predictors of health-related quality of life in boys with Duchenne muscular dystrophy from six European countries. Journal of Neurology 2017; 264: 709-23. https://doi.org/10.1007/s00415-017-8406-2.

[20] Wei Y, Speechley KN, Zou G, Campbell C. Factors associated with health-related quality of life in children with Duchenne muscular dystrophy. Journal of Child Neurology 2016; 31: 879-86. https://doi.org/10.1177/0883073815627879.

[21] Abresch RT, Carter GT, Jensen MP, Kilmer DD. Assessment of pain and health-related quality of life in slowly progressive neuromuscular disease. American Journal of Hospice and Palliative Medicine 2002; 19: 39-48.

[22] Kalkman JS. Experienced fatigue in facioscapulohumeral dystrophy, myotonic dystrophy, and HMSN-I. Journal of Neurology, Neurosurgery \& Psychiatry 2005; 76: 1406-09. https://doi.org/10.1136/jnnp.2004.050005.

[23] Paschalides C, Wearden AJ, Dunkerley R, Bundy C, Davies R, Dickens CM. The associations of anxiety, depression and personal illness representations with glycaemic control and healthrelated quality of life in patients with type 2 diabetes mellitus. Journal of Psychosomatic Research 2004; 57: 557-64. https://doi.org/10.1016/j.jpsychores.2004.03.006.

[24] Goldney RD, Phillips PJ, Fisher U, Wilson DH. Diabetes, depression, and quality of life: A population study. Diabetes Care 2004; 27: 1066-70. 
[25] Wexler DJ, Grant RW, Wittenberg E, Bosch JL, Cagliero E, Delahanty L, et al. Correlates of health related quality of life in type 2 diabetes. Diabetologia 2006; 49: 1489 -97. https://doi.org/10.1007/s00125-006-0249-9.

[26] Verma SK, Luo N, Subramaniam M, Sum CF, Stahl D, Liow PH, et al. Impact of depression on health related quality of life in patients with diabetes. Annals of the Academy of Medicine 2010; 39: 913-19.

[27] Pangalila RF, van den Bos GA, Bartels B, Bergen M, Stam HJ, Roebroeck ME. Prevalence of fatigue, pain, and affective disorders in adults with Duchenne muscular dystrophy and their associations with quality of life. Archives of Physical Medicine and Rehabilitation 2015; 96 : 1242-47. https://doi.org/10.1016/j.apmr.2015.02.012.

[28] Mann MM, Hosman CM, Schaalma HP, De Vries NK. Self-esteem in a broad-sprectrum approach for mental health promotion. Health Education Research 2004; 19: 357-72. https://doi.org/10.1093/her/cyg041.

[29] Rosenberg M, Schooler C, Schoenbach C, Rosenberg F. Global self-esteem and specific self-esteem: Different concepts, different outcomes. American Sociological Review 1995; 60: 141-56.

[30] Mikula P, Nagyova I, Krokavcova M, Vitkova M, Rosenberger J, Szilasiova J, et al. Selfesteem, social participation, and quality of life in patients with multiple sclerosis. Journal of Health Psychology 2017; 22: 984-92. https://doi.org/10.1177/1359105315621778.

[31] Bartoces MG, Severson RK, Rusin RA, Schwartz KL, Ruterbusch JL, Neale AV. Quality of life and self-esteem of long-term survivors of invasive and noninvasive cervical cancer. Journal of Women's Health 2009; 18: 655-61. https://doi.org/10.1089/jwh.2008.0959.

[32] Garcia-Martinez AM, De Paz JA, Marquez S. Effects of an exercise programme on selfesteem, self-concept and quality of life in women with fibromyalgia: a randomized controlled trial. Rheumatology International 2012; 32: 1869-76. https://doi.org/10.1007/s00296-0111892-0.

[33] McDonald CM, Henricson EK, Han JJ, Abresch RT, Nicorici A, Elfring GL, et al. The 6-minute walk test as a new outcome measure in Duchenne muscular dystrophy. Muscle \& Nerve 2010; 41: 500-10. https://doi.org/10.1002/mus.21544.

[34] Bostock EL, O'Dowd DN, Payton CJ, Smith D, Orme P, Edwards BT, et al. The effects of resistance exercise training on strength and functional tasks in adults with Limb-Girdle, Becker and Facioscapulohumeral Dystrophies. Frontiers in Neurology 2019; 10: 1216.

[35] American Thoracic Society. ATS statement: guidelines for the six-minute walk test. American Journal of Respiratory and Critical Care Medicine 2002; 166: 111-17.

[36] Ware JE. SF-36 Health Survey update. Spine 2000; 25: 3130-39.

[37] Sprinkle SD, Laurie D, Insko SL, Atkinson G, Jones GL, Logan AR, et al. Criterion validity, severity cut scores, and test-retest reliability of the Beck Depression Inventory-II in a 
university counseling center sample. Journal of Counseling Psychology 2002; 49: 381-85. https://doi.org/10.1037//0022-0167.49.3.381.

[38] Spielberger CD, Gorsuch RL, Lushene RE, Vagg PR, Jacobs GA. Manual for the State-Trait Anxiety Inventory STAI (Form Y). Palo Alto: Consulting Psychologists Press; 1983.

[39] Rosenberg M. Society and the adolescent self-image. New Jersey: Princeton University Press; 1965.

[40] Sinclair SJ, Blais MA, Gansler DA, Sandberg E, BIstis K, LoCicero A. Psychometric properties of the Rosenberg Self-Esteem Scale: Overall and across demographic groups living within the United States. Evaluation \& the Health Professions 2010; 33: 56-80. https://doi.org/10.1177/0163278709356187.

[41] Fox KR, Corbin CB. The-Physical-Self-Perception Profile: Development and preliminary validation. Journal of Sport and Exercise Psychology 1989; 11: 408-30. https://doi.org/10.1123/jsep.11.4.408.

[42] Mukaka MM. A guide to appropriate use of correlation coefficient in medical research. Malawi Medical Journal 2012; 23: 69-71.

[43] Raustorp A, Stahle A, Gudasic H, Kinnunen A, Mattson E. Physical activity and selfperception in school children assessed with the Children and Youth - Physical Self-Perception Profile. Scandinavian Journal of Medicine \& Science in Sports 2005; 15: 126-34. https://doi.org/10.1111/j.1600-0838.2004.406.x. 
Table 1: Participant characteristics. Maximum voluntary contraction torque is expressed as a percentage of the control group mean value.

\begin{tabular}{lccccc}
\hline & MD & BMD & FSHD & LGMD & CTRL \\
\hline $\mathrm{n}$ & 21 & 7 & 8 & 6 & 10 \\
Age (years) & $45.4 \pm 10.5$ & $42.1 \pm 7.6$ & $46.9 \pm 12.6$ & $47.3 \pm 11.3$ & $46.8 \pm 10.2$ \\
Stature (cm) & $177.4 \pm 8.6$ & $179.9 \pm 9.4$ & $180.0 \pm 4.9$ & $170.9 \pm 9.0$ & $174.4 \pm 7.5$ \\
Mass (kg) & $86.9 \pm 17.4$ & $93.0 \pm 16.7$ & $85.3 \pm 17.3$ & $82.0 \pm 19.4$ & $79.4 \pm 12.3$ \\
KF MVC (\%) & $37 \pm 31$ & $27 \pm 15$ & $53 \pm 43$ & $25 \pm 15$ & $100 \pm 28$ \\
KE MVC (\%) & $33 \pm 34$ & $11 \pm 7$ & $59 \pm 42$ & $21 \pm 18$ & $100 \pm 38$ \\
Unemployed (\%) & 9 & 14 & 13 & 0 & 0 \\
Employed (\%) & 67 & 57 & 62 & 83 & 20 \\
\hline Retired (\%) & 24 & 29 & 25 & 17 & \\
\hline
\end{tabular}

MD: muscular dystrophy; BMD: Becker; FSHD: Facioscapulohumeral; LGMD: Limb-girdle; CTRL: control; KF MVC: knee flexion maximum voluntary contraction torque: KE MVC: knee extension maximum voluntary contraction torque. 
Table 2: Quality of life scores in each group. Maximum score in each QoL domain is 100.

\begin{tabular}{|c|c|c|c|c|c|}
\hline & MD & BMD & FSHD & LGMD & CTRL \\
\hline Physical Function & $22 \pm 17^{* * *}$ & $18 \pm 10^{* * *}$ & $26 \pm 21^{* * *}$ & $22 \pm 17^{* * *}$ & $98 \pm 4$ \\
\hline Role Physical & $54 \pm 27^{* * *}$ & $46 \pm 29^{* * *}$ & $61 \pm 20^{* * *}$ & $54 \pm 35^{* * *}$ & $99 \pm 4$ \\
\hline Bodily Pain & $58 \pm 29^{* *}$ & $57 \pm 35$ & $52 \pm 29^{* *}$ & $67 \pm 22^{*}$ & $89 \pm 10$ \\
\hline General Health & $53 \pm 21^{* *}$ & $55 \pm 20^{*}$ & $56 \pm 25^{*}$ & $48 \pm 19^{* *}$ & $77 \pm 15$ \\
\hline Vitality & $47 \pm 21^{* * *}$ & $47 \pm 27^{*}$ & $51 \pm 21^{* *}$ & $42 \pm 13^{* *}$ & $71 \pm 8$ \\
\hline Social Function & $64 \pm 28^{* * *}$ & $57 \pm 33^{* * *}$ & $67 \pm 30^{* *}$ & $69 \pm 21^{* *}$ & $99 \pm 4$ \\
\hline Role Emotion & $77 \pm 25^{* *}$ & $76 \pm 28^{*}$ & $73 \pm 28^{*}$ & $82 \pm 21^{*}$ & $98 \pm 3$ \\
\hline Mental Health & $71 \pm 18$ & $74 \pm 18$ & $71 \pm 23$ & $70 \pm 14$ & $84 \pm 6$ \\
\hline PCS & $35 \pm 8^{* * *}$ & $33 \pm 10^{* * *}$ & $37 \pm 5^{* * *}$ & $35 \pm 10^{* * *}$ & $57 \pm 3$ \\
\hline MCS & $52 \pm 12$ & $52 \pm 14$ & $51 \pm 13$ & $52 \pm 8$ & $55 \pm 3$ \\
\hline
\end{tabular}

MD: muscular dystrophy, BMD: Becker, FSHD: Facioscapulohumeral, LGMD: Limb-girdle, CTRL: control, PCS: Physical Component Score,

MCS: Mental Component Score. ${ }^{*}$ denotes significant difference from control $p<.05,{ }^{* *}$ denotes significant difference from control $p \leq$ $.010,{ }^{* * *}$ denotes significant difference from control $p \leq .001$. 
Table 3: Psychological parameters and 6MW distance in each group. Maximum score for each parameter is indicated in the $1^{\text {st }}$ column.

\begin{tabular}{lccccc}
\hline & MD & BMD & FSHD & LGMD & CTRL \\
\hline DS /63 & $10.9 \pm 7.9^{* *}$ & $11.3 \pm 10.5$ & $10.9 \pm 7.9$ & $10.3 \pm 5.6$ & $4.2 \pm 3.0$ \\
TA /80 & $39.2 \pm 11.4$ & $39.9 \pm 13.1$ & $38.6 \pm 11.6$ & $39.2 \pm 11.1$ & $32.6 \pm 8.4$ \\
SE /40 & $20.9 \pm 5.6$ & $21.0 \pm 5.4$ & $21.8 \pm 6.3$ & $19.5 \pm 5.7$ & $24.3 \pm 5.2$ \\
PSW /24 & $10.8 \pm 3.3^{* * *}$ & $10.8 \pm 3.8^{*}$ & $11.5 \pm 3.7^{*}$ & $9.8 \pm 2.6^{* * *}$ & $16.7 \pm 3.9$ \\
6MWD (m) & $333 \pm 112^{* * *}$ & $321 \pm 77^{* *}$ & $397 \pm 102$ & $276 \pm 122^{* *}$ & $481 \pm 72$ \\
\hline
\end{tabular}

MD: muscular dystrophy, BMD: Becker, FSHD: Facioscapulohumeral, LGMD Limb-girdle, CTRL: control. DS: depressive symptom score, TA:

trait anxiety score, SE: self-esteem score, PSW: physical self-worth score, 6MWD: six-minute walk distance. ${ }^{*}$ denotes significant difference

from control $p<.05, * *$ denotes significant difference from control $p \leq .010, * * *$ denotes significant difference from control $p \leq .001$. 
Table 4: Associations between QoL and psychological parameters/6MW distance.

\begin{tabular}{|c|c|c|c|c|c|}
\hline & DS & TA & SE & PSW & 6MWD \\
\hline Physical Function & - & - & - & - & $.647^{* *}$ \\
\hline Role Physical & - & $-.442^{*}$ & - & $.490^{*}$ & - \\
\hline Bodily Pain & $-.450^{*}$ & - & - & - & - \\
\hline General Health & $-.434^{*}$ & - & - & $.500^{*}$ & - \\
\hline Vitality & $-.445^{*}$ & $-.482^{*}$ & - & $.518^{*}$ & $.511^{*}$ \\
\hline Social Function & $-.664^{* * *}$ & $-.744^{* * *}$ & $.466^{*}$ & - & - \\
\hline Role Emotion & $-.783^{* * *}$ & $-.854^{* * *}$ & $.680^{* * *}$ & - & - \\
\hline Mental Health & $-.894^{* * *}$ & $-.811^{* * *}$ & $.765^{* * *}$ & $.584^{* *}$ & - \\
\hline PCS & - & - & - & - & $.640^{* *}$ \\
\hline MCS & $-.874^{* * *}$ & $-.822^{* * *}$ & $.743^{* * *}$ & $.554^{*}$ & - \\
\hline
\end{tabular}

DS: depressive symptoms, TA: trait anxiety, SE: self-esteem, PSW: physical self-worth, 6MWD six-minute walk distance. PCS: Physical Component Score, MCS: Mental Component Score. - denotes non-significant relationships, ${ }^{*}$ denotes significant difference $\mathrm{p}<.05, * *$ denotes significant difference $p \leq .010, * * *$ denotes significant difference $p \leq .001$. 